\title{
EFEITO DA IDADE SOBRE AS PERDAS ENDÓGENAS E METABÓLICAS DE FRANGOS DE CORTE INDUSTRIAL E CAIPIRA
}

\author{
Edney Pereira da Silva, ${ }^{1}$ Carlos BôA-Viagem Rabello, ${ }^{2}$ Michele Bernardino de Lima, ${ }^{3}$ \\ Stélio Bezerra Pinheiro de Lima, ${ }^{4}$ Rodrigo Barbosa Lima ${ }^{4}$ e Tayara Soares de Lima ${ }^{5}$ \\ 1. Doutorando em Zootecnia na UNESP/Campus Jaboticabal. E-mail: edneysilva@oi.com.br \\ 2. Professor adjunto, Departamento de Zootecnia, Universidade Federal Rural de Pernambuco \\ 3. Aluna de graduação em Zootecnia, bolsista do PIBIC/CNPq, DZ/UFRPE \\ 4. Aluno do Programa de Doutorado Integrado em Zootecnia PDIZ/UFRP \\ 5. Aluna de graduação em Zootecnia, bolsista do PET/MEC/SESu/DZ/UFRPE
}

RESUMO

O objetivo deste estudo foi determinar as perdas endógenas e metabólicas em frangos de corte industrial e caipira em diferentes idades. No primeiro experimento, utilizaram-se 140 pintos de corte industrial, distribuídos num delineamento inteiramente casualizado, com quatro tratamentos e cinco repetições. No segundo experimento, foram utilizadas 120 aves de sexo misto, da linhagem caipira Embrapa 041, submetidos a três tratamentos e cinco repetições distribuídas num mesmo delineamento. Em ambos os experimentos os tratamentos tiveram como parâmetro as idades médias das aves. Para o experimento 1 as idades foram $5,15,25$ e 35 dias de nascimento; para o experimento 2 as idades médias adotadas foram 15, 45 e 75 dias de nascimento. O período experimental foi de 72 horas de jejum, sendo as primeiras 24 horas para limpeza do trato digestivo e as últimas 48 horas de coleta total das perdas endógenas e metabólicas. Foram determinados os teores de matéria seca, nitrogênio e energia bruta das perdas endógenas e metabólicas das aves. Ao se relacionarem as perdas, por ave e peso da ave, diferentes interpretações foram obtidas para a mesma variável. As equações obtidas para os frangos industrial e caipira revelaram que essas aves tiveram comportamentos diferentes na excreção fecal metabólica e urinária endógena. As perdas diminuíram com o avanço da idade.

PALAVRAS-CHAVES: Aves em jejum, método de coleta total, energia fecal metabólica, energia urinária endógena.

\section{ABSTRACT}

\section{AGE EFFECT ON THE ENDOGENOUS AND METABOLIC LOSSES IN BROILER CHICKENS AND FREE RANGE}

The objective of this study was to determine the endogenous and metabolic losses in broiler chickens and free range on different age rates. On the experiment 1,140 broiler chickens distributed in an entirely randomized design with four treatments and five replications have been used. On the second experiment, 120 birds of mixed sex from the Embrapa 041 free range line distributed in tree treatments and five replications distributed in a same design. On both experiments the treatments consisted in the average ages of the birds. To the first experiment the ages were: $5 ; 15 ; 25$ and 35 days of age and the second experiment the average ages adopted were 15 ,
45 and 75 days of age. The experimental period was of 72 hours of fasting, being the first 24 hours for digestive tract cleaning and the last 48 hours for total collection of the endogenous and metabolic losses. The contents of dry matter, nitrogen and brute energy from birds` endogenous and metabolic losses have been determined. When relating losses per bird and bird weight, different interpretations were obtained for the same variable. The equations obtained to broiler chicken and free range reveled that those birds had different behaviors during metabolic fecal and endogenous urinary. The losses diminished according to age advance.

KEYWORDS: Birds on fasting, total collection method, metabolic fecal energy, endogenous urinary energy. 


\section{INTRODUÇÃO}

Há mais de três décadas pesquisadores estudam as perdas endógenas e metabólicas e seus respectivos efeitos sobre a determinação dos valores energéticos dos alimentos para aves. Nesse período, aprimoraram-se o conceito, e a padronização das metodologias, a fim de aumentar a precisão na determinação real de energia aproveitada pelos animais.

Conceitualmente, de forma detalhada, nas fezes das aves encontram-se os resíduos não digeridos e a fração metabólica formada por bílis, secreções digestivas e células procedentes da mucosa intestinal e nas aves não cecectomizadas à ação da microbiota. Na fração urinária predominam os resíduos alimentares absorvidos e eliminados sem ser catabolizados, adicionados da fração endógena, que consiste no produto do catabolismo dos tecidos (SIBBALD \& PRICE, 1978; SIBBALD, 1981a; SILVA et al., 2006).

O sistema de energia metabolizável aparente (EMA) supõe que toda energia excretada é proveniente do alimento, o que não é verdade. Motivado por essa premissa, SIBBALD (1976) propôs a correção das perdas endógenas e metabólicas nos valores de EMA, sugerindo que o sistema de energia metabolizável verdadeira (EMV) representaria melhor a energia do alimento.

O referido autor afirmou que as frações endógenas e metabólicas presentes na excreta das aves em regime ad libitum são semelhantes às frações coletadas em aves submetidas ao jejum. Essa afirmação também não é verdadeira, e tem motivado discussões no campo teórico entre os pesquisadores.

Assim, na literatura existem relatos de que o sistema de EMV expressa com mais segurança o conteúdo energético dos alimentos para aves, quando comparado ao sistema de EMA(SIBBALD, 1976; DALE \& FULLER, 1982; BORGES et al., 2003). Contudo, essa premissa não tem sido confirmada em alguns trabalhos de desempenho animal, como o de FREITAS et al. (2006), que verificaram maiores consumo de ração, ganho de peso e melhor conversão alimentar com o sistema de EMA.

Hoje, sabe-se que dentre os fatores que afetam a intensidade das perdas endógenas e metabólicas em aves estão: a metodologia aplicada, o regime alimentar, a genética, a categoria (BORGES et al., 2003) e, para frangos industriais, como descoberto recentemente, especificamente a idade (SILVA et al., 2006). Dessa maneira, o objetivo deste trabalho de pesquisa foi determinar o efeito da idade sobre as perdas endógenas e metabólicas em frangos de corte industrial e caipira.

\section{MATERIAL E MÉTODOS}

Realizaram-se dois experimentos: um com frango de corte industrial e outro com frango de corte caipira. $\mathrm{O}$ primeiro experimento foi feito no Departamento de Zootecnia da Universidade Federal Rural de Pernambuco (UFRPE), com 140 pintos de corte machos da linhagem Cobb, de um dia de idade. As aves foram distribuídas em um delineamento experimental inteiramente casualizado, com quatro tratamentos e cinco repetições.

Os tratamentos consistiram em quatro idades médias de frango de corte $(5,15,25$ e 35 dias de idade), sendo 10, 8, 6 e 4 o número de aves por parcela experimental nas respectivas idades. Os pesos médios das aves no início do período experimental foram 83,28 g (T1); 434, $10 \mathrm{~g}$ (T2); 1.004,80 g (T3) e 2.310,60 g (T4). As aves foram selecionadas pelo peso, buscando a máxima uniformidade, e alojadas em gaiolas de metabolismo dotadas de sistema de aquecimento na primeira semana de vida e de bandejas coletoras sob o piso.

Inicialmente, transferiram-se as aves para as baterias $(1,00 \times 0,50 \times 0,50 \mathrm{~m}) \operatorname{nos} 1^{\circ}, 10^{\circ}, 20^{\circ}$ e $30^{\circ}$ dias de idade, obedecendo-se a um período de 4 dias de adaptação às instalações. As aves foram mantidas em jejum a partir dos $4^{\circ}, 14^{\circ}, 24^{\circ}$ e $34^{\circ}$ dias por um período de 24 horas para esvaziar o trato digestório e, em seguida, por mais 48 horas, para coleta do material, resultando em idades médias experimentais ao $5^{\circ}$ dia de idade (T1), $15^{\circ}$ dia de idade (T2), $25^{\circ}$ dia de idade (T3) e $35^{\circ}$ dia de idade (T4), como já relatado.

As aves utilizadas foram descartadas ao final de cada experimento. Assim, de um total de 240, para cada idade selecionaram-se novas aves que foram alojadas no primeiro dia de vida em boxes dotados de lâmpadas incandescentes de 100 watts para aquecimento nas duas primeiras semanas de vida. O material utilizado como cama foi maravalha. As aves foram arraçoadas com uma dieta à base de milho e farelo de soja, formulada para atender às exigências nutricionais nas diferentes idades, segundo ROSTAGNO et al. (2005).

Durante o experimento, após o jejum de 24 horas em cada idade, fez-se duas vezes ao dia a coleta dos resíduos excretados (material endógeno e metabólico) 
pelas aves por um período de 48 horas. Em seguida, o material coletado de cada parcela experimental foi acondicionado e congelado em freezer a $-20^{\circ} \mathrm{C}$.

Ao término do experimento, o material foi descongelado, homogeneizado, amostrado, pesado e pré-seco em estufa de ventilação forçada regulada para $55^{\circ} \mathrm{C}$ por 72 horas. No fim da pré-secagem, as excretas foram moídas em moinho de facas, dotado de peneira de $1 \mathrm{~mm}$ de abertura de crivo, para serem feitas as análises de matéria seca, nitrogênio e energia bruta, seguindo as metodologias descritas por SILVA \& QUEIROZ (2002).

Após as análises, calcularam-se a matéria seca fecal metabólica e urinária endógena (MSfm + MSue), o nitrogênio fecal metabólico e urinário endógeno ( $\mathrm{Nfm}$ + Nue) e a energia fecal metabólica e urinária endógena (Efm+Eue), além das perdas endógenas e metabólicas de matéria seca e nitrogênio.

O segundo experimento foi conduzido na Estação Experimental de Pequenos Animais de Carpina (EEPAC), pertencente à UFRPE. Utilizaram-se 120 aves de sexo misto da linhagem caipira Embrapa 041. As aves foram distribuídas num delineamento experimental inteiramente casualizado, com três tratamentos e cinco repetições. Os tratamentos tiveram como parâmetros as idades médias dos frangos caipiras: 15, 45 e 75 dias de idade, utilizando dez, oito e seis aves por parcela experimental nas respectivas idades, sendo metade de cada sexo.

As aves foram transferidas para as baterias $(1,00 \times 0,50 \times 0,50 \mathrm{~m})$ aos $10^{\circ}, 40^{\circ}$ e $70^{\circ}$ dias de idade e obedeceu-se a um período de 4 dias de adaptação às instalações. As aves foram mantidas em jejum aos $14^{\circ}, 44^{\circ}$ e $74^{\circ}$ dias por 24 horas, para limpar todo trato digestório; em seguida houve jejum por mais 48 horas, para coleta do material, resultando em idades médias experimentais aos 14 dias de idade (T1), 45 dias de idade (T2) e 75 dias de idade (T3).

No período pré-experimental, as aves foram criadas em galpão convencional, com fornecimento de ração e água à vontade. A ração ofertada era à base de milho e farelo de soja, formulada de acordo com as exigências nutricionais para as diferentes idades, como preconizado pelo manual da linhagem (Tabela 1).

TABELA 1. Composição percentual e valores nutricionais das rações

\begin{tabular}{|c|c|c|c|c|c|c|c|}
\hline \multirow{2}{*}{ Ingredientes } & \multicolumn{4}{|c|}{ Frango de corte industrial } & \multicolumn{3}{|c|}{ Frango de corte caipira } \\
\hline & Pré-inicial & Inicial & Crescimento & Final & Inicial & Crescimento & Final \\
\hline Milho grão & 56,650 & 59,657 & 62,557 & 66,556 & 61,625 & 66,300 & 70,300 \\
\hline Farelo de soja $45 \%$ & 36,768 & 34,227 & 30,618 & 26,779 & 31,905 & 26,270 & 22,090 \\
\hline Farelo de trigo & - & - & - & - & 2,548 & 3,611 & 4,104 \\
\hline Óleo de soja & 2,048 & 2,183 & 3,097 & 3,120 & - & - & - \\
\hline Fosfato bicálcio & 1,943 & 1,798 & 1,653 & 1,506 & 1,910 & 1,750 & 1,495 \\
\hline Calcário calcítico & 0,939 & 0,894 & 0,851 & 0,807 & 1,128 & 1,259 & 1,311 \\
\hline Sal comum & 0,517 & 0,492 & 0,469 & 0,442 & 0,508 & 0,457 & 0,418 \\
\hline DL-metionina $99 \%$ & 0,445 & 0,250 & 0,240 & 0,230 & 0,086 & 0,093 & 0,082 \\
\hline L-lisina $\mathrm{HCl}$ & 0,400 & 0,219 & 0,235 & 0,280 & - & - & - \\
\hline Suplemento vitamínico & $0,100^{1}$ & $0,100^{1}$ & $0,100^{1}$ & $0,100^{1}$ & $0,100^{2}$ & $0,080^{2}$ & $0,040^{2}$ \\
\hline Suplemento mineral ${ }^{2}$ & $0,050^{3}$ & $0,050^{3}$ & $0,050^{3}$ & $0,050^{3}$ & $0,050^{4}$ & $0,050^{4}$ & $0,050^{4}$ \\
\hline Anticoccidiano & 0,050 & 0,050 & 0,050 & 0,050 & 0,050 & 0,050 & 0,050 \\
\hline Bacitracina de zinco $15 \%$ & 0,040 & 0,040 & 0,040 & 0,040 & 0,040 & 0,040 & 0,040 \\
\hline Cloreto de colina $60 \%$ & 0,050 & 0,040 & 0,040 & 0,040 & 0,050 & 0,040 & 0,020 \\
\hline Total & 100,00 & 100,00 & 100,00 & 100,00 & 100,00 & 100,00 & 100,00 \\
\hline EMAn, Mcal $/ \mathrm{kg}$ & 2,960 & 3,000 & 3,100 & 3,150 & 2,850 & 2,900 & 2,950 \\
\hline Proteína bruta, \% & 22,11 & 20,79 & 19,41 & 18,03 & 20,00 & 18,00 & 16,50 \\
\hline Lisina total, $\%$ & 1,503 & 1,263 & 1,183 & 1,121 & 1,044 & 0,904 & 0,800 \\
\hline Met + cistina total, $\%$ & 1,067 & 0,897 & 0,852 & 0,807 & 0,724 & 0,681 & 0,633 \\
\hline Metionina total, $\%$ & 0,728 & 0,568 & 0,540 & 0,512 & 0,400 & 0,380 & 0,350 \\
\hline Treonina total, $\%$ & 0,836 & 0,800 & 0,745 & 0,690 & 0,770 & 0,690 & 0,630 \\
\hline Cálcio, \% & 0,942 & 0,884 & 0,824 & 0,763 & 1,000 & 1,000 & 0,950 \\
\hline Fósforo disponível, \% & 0,471 & 0,442 & 0,411 & 0,380 & 0,468 & 0,436 & 0,386 \\
\hline Sódio, \% & 0,224 & 0,214 & 0,205 & 0,194 & 0,220 & 0,200 & 0,190 \\
\hline
\end{tabular}

1 Níveis de garantia por kg de produto: vit. A, 6.000.000 UI, vit. D3 1.000.000 UI, vit. E $10.000 \mathrm{mg}$, vit. B12 6.000 mcg, vit. K3 1.000, niacina 10.000 $\mathrm{mg}$, piridoxina $800 \mathrm{mg}$, riboflavina $2.000 \mathrm{mg}$, tiamina $600 \mathrm{mg}$, biotina $30 \mathrm{mg}$, pantotenato de cálcio $8.000 \mathrm{mg}$, selênio $400 \mathrm{mg}$. ${ }^{2} \mathrm{Quantidade} / \mathrm{kg}$ de ração: : vit. A, 11.000 U.I.; vit. D3 2.000 UI.; vit. E 16 UI.; ácido fólico 0,4 mg; pantotenato de cálcio 10,0 mg; biotina 0,06 mg; niacina $35 \mathrm{mg}$; piridoxina 2,0 $\mathrm{mg}$; riboflavina 4,5 mg; tiamina 1,2 mg; vit. B12 $16,0 \mathrm{mg}$; vit. K3 $1,5 \mathrm{mg}$; selênio $0,25 \mathrm{mg}$; antioxidante $30 \mathrm{mg}$.

${ }^{3}$ Níveis de garantia por kg de produto: cobre: $18.000 \mathrm{mg}$, zinco: $120.000 \mathrm{mg}$, iodo: $2.000 \mathrm{mg}$, ferro: $60.000 \mathrm{mg}$, manganês: $120.000 \mathrm{mg}$. ${ }^{4} \mathrm{Quantidade} /$ kg de ração: cobre 9,0 mg; zinco 60,0 mg; manganês 60,0 mg; ferro 30,0 mg; iodo 1,0 mg. 
A seleção dos animais foi baseada no peso médio das aves, buscando a uniformidade da parcela. Os pesos médios foram $0,273 \mathrm{~g}, 0,921 \mathrm{~g}$ e $2,047 \mathrm{~g}$ para as idades de 15, 45 e 75 dias, respectivamente. Em ambos os experimentos forneceu-se água à vontade durante todo o período. A coleta, manipulação, análise do material excretado e os cálculos dos parâmetros interessados foram os mesmos citados no primeiro experimento.

As variáveis dependentes analisadas foram: perdas endógenas e metabólicas de matéria seca, nitrogênio e energia bruta, nas diferentes idades, expressas em diferentes sistemas de unidade. Os resultados obtidos foram submetidos à análise de regressão, utilizando-se o programa computacional SISVAR versão 4.6 (FERREIRA, 2003).

\section{RESULTADOS E DISCUSSÃO}

A composição química das perdas endógenas e metabólicas de frangos de corte industrial e caipira está apresentada na Tabela 2. Os resultados encontrados revelaram que a maior concentração de matéria seca (MS) foi verificada a partir dos 25 dias de idade, enquanto a menor concentração encontrava-se nas aves com idade média de 5 dias. Diferentemente da MS, a energia bruta (EB) das perdas endógenas e metabólicas não teve alterações nas diferentes idades; a análise de nitrogênio $(\mathrm{N})$ apresentou o menor teor aos 25 dias de idade, não existindo diferenças entre as demais idades.

As perdas endógenas e metabólicas dos frangos de corte caipira tiveram as maiores concentrações de matéria seca e energia bruta aos 45 dias de idade. Nessa idade a concentração de nitrogênio apresentou o menor teor, não existindo diferenças entre as idades equidistantes (15 e 75 dias). Fatores relacionados a jejum, idade, composição corporal, genótipo e estado fisiológico da ave são relatados (SIBBALD, 1981b; SILVA et al., 2006) como condicionantes para a composição química das perdas endógenas e metabólicas. Assim, a composição química deve ser relacionada a outras variáveis, para que faça a contextualização adequada e para que haja interpretações conclusivas e coerentes sobre os acontecimentos pelos quais a ave passou em estado de jejum e em jejum prolongado.

TABELA 2. Médias de composição química e energética das perdas endógenas e metabólicas expressas com base na matéria seca

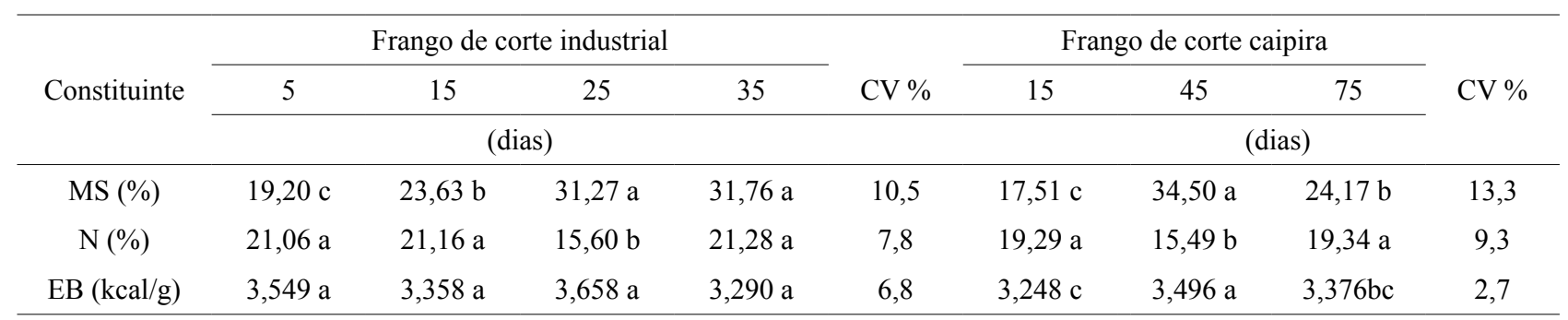

a,b,c Médias seguidas de mesma letra na linha diferem estatisticamente pelo teste Student Newman-Keuls a $5 \%$ de probabilidade. $\mathrm{CV}=$ coeficiente de variação \%.

Ao se relacionar a matéria seca fecal metabólica e urinária endógena com o volume excretado por parcela, por ave e por peso da ave, nota-se que as diferenças entre as idades existem $(\mathrm{p} \leq 0,01)$; contudo, conforme o sistema em que ela está sendo expressa, os resultados revelam comportamentos opostos (Tabela 3 ).

Ao se fazer a relação com o peso corporal da ave, observou-se um resultado oposto. Assim, as aves com a idade média de 5 dias excretaram uma quantidade equivalente a $8 \%$ do seu peso. As diferenças se anularam a partir do $15^{\circ}$ dia de vida e ao $35^{\circ}$ dia a perda correspondeu a pouco mais de um décimo do peso corporal da ave.

O ajuste do metabolismo às diferentes condições fisiológicas é obtido graças aos processos que compõem a regulação metabólica. Assim, 12 horas após a refeição inicia-se o estado pós-absortivo; passado esse momento, a degradação de glicogênio, a proteólise muscular e a lipólise se sucedem, sendo responsáveis por manter o aporte energético no organismo (MALHEIROS, 2008). 
TABELA 3. Médias e erros-padrões, equação de regressão, probabilidade e coeficiente de variação para os valores das perdas de matéria seca fecal metabólica e urinária endógena de frango de corte industrial em diferentes idades

\begin{tabular}{ccccc}
\hline \multirow{2}{*}{$\begin{array}{c}\text { Idade } \\
\text { (dias) }\end{array}$} & \multicolumn{5}{c}{} \\
\cline { 2 - 5 } & (gMS/ave) & $(\mathrm{gMS} / \mathrm{kgPC})$ & $(\% \mathrm{de} \mathrm{PC})$ & $(\mathrm{gMS} / \mathrm{kgPC}, 75)$ \\
\hline 5 & $6,00 \mathrm{~d}$ & $72,38 \mathrm{a}$ & $8,76 \mathrm{a}$ & $69,05 \mathrm{a}$ \\
15 & $10,73 \mathrm{c}$ & $24,74 \mathrm{~b}$ & $0,71 \mathrm{~b}$ & $33,76 \mathrm{~b}$ \\
25 & $15,10 \mathrm{~b}$ & $15,02 \mathrm{bc}$ & $0,25 \mathrm{~b}$ & $23,54 \mathrm{bc}$ \\
35 & $23,29 \mathrm{a}$ & $10,09 \mathrm{c}$ & $0,11 \mathrm{~b}$ & $17,58 \mathrm{c}$ \\
Média $^{1}$ & $13,78 \pm 0,62$ & $30,56 \pm 3,96$ & $2,46 \pm 0,53$ & $35,98 \pm 3,79$ \\
$\mathrm{ER}^{1}$ & $\hat{\mathrm{Y}}=2,530+0,563 \mathrm{X}$ & $\hat{\mathrm{Y}}=69,877-1,967 \mathrm{X}$ & $\hat{\mathrm{Y}}=7,743-0,264 \mathrm{X}$ & $\hat{\mathrm{Y}}=68,903-1,646 \mathrm{X}$ \\
$\mathrm{R}^{2}, \%$ & 97,62 & 79,09 & 65,68 & 85,12 \\
$\mathrm{P}^{2}$ & 0,0001 & 0,0001 & 0,0001 & 0,0001 \\
$\mathrm{CV}^{3}$ & 10,19 & 29,03 & 47,97 & 23,57 \\
\hline
\end{tabular}

a,b,c,d Médias seguidas de letras diferentes, na coluna, diferem estatisticamente pelo teste Student Newman-Keuls a 1\% de probabilidade.

'Equação de regressão.

${ }^{2}$ Probabilidade.

${ }^{3}$ Coeficiente de variação, $\%$.

No momento de jejum, o glucagon é favorecido na relação insulina-glucagon, e a glicogenólise é intensificada. Já no jejum prolongado, o índice do glicêmico é mantido pela gliconeogênese, relacionada à perda significativa de massa muscular e de tecido adiposo que acompanham o jejum (NELSON \& COX, 2000).

De forma prática, a relação entre o metabolismo basal e o peso metabólico possibilita justificar e interpretar mais coerentemente os eventos fisiológicos ocorridos na ave, sobretudo quando se compara a taxa metabólica de animais com pesos e composição corporais diferentes, conforme BLAXTER (1989).

Assim, a grama de matéria seca fecal metabólica e urinária endógena (MSfm + MSeu) excretada por ave aumentou com o avançar da idade de forma linear $\hat{Y}=2,530+0,563 X, R^{2}=97,62$ (Figura 1A). Contrariamente quando se relacionou a excreção de MSfm + MSeu ao peso metabólico da ave, a excreção diminuiu cerca de 1,646 g/dia, conforme a equação $\hat{Y}$ $=68,903-1,646 \mathrm{X}, \mathrm{R}^{2}=85,12$ (Figura 1B).

Nessa fase são marcantes as diferenças nos constituintes químicos corporais da ave (SILVA et al., 2006), e esses fatores são responsáveis pelas diferenças apresentadas, uma vez que a composição corporal se relaciona intimamente com a expressão e a intensidade das perdas endógenas e metabólicas, segundo os autores supracitados.
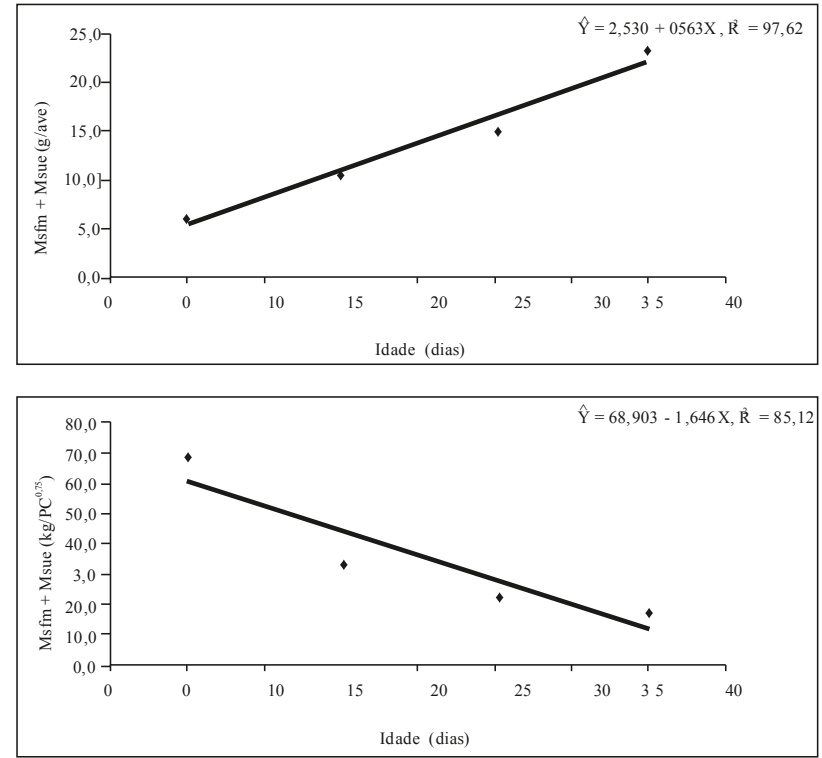

FIGURA 1. Efeito da idade sobre as perdas em matéria seca fecal metabólica e urinária endógena (MSfm + MSeu) de frangos de corte industrial expresso por ave (A) e expresso em peso metabólico (B).

A Tabela 4 mostra diferenças marcantes na fase inicial das aves de crescimento lento, em que a excreção MSfm + MSeu por ave foi aproximadamente 2,4 vezes menor que a excreção nas fases subsequentes, crescimento e final. Na fase inicial, comparativamente, 4,25 g de matéria seca faz diferir o quantitativo 
expelido pelas aves de corte industrial e pelas aves de corte caipira. Em relação percentual, as aves de corte industrial perdem no mesmo período de coleta cerca de $70 \%$ a mais que as aves caipiras.

TABELA 4. Médias e erros-padrões, equação de regressão, probabilidade e coeficiente de variação para os valores das perdas de matéria seca fecal metabólica e urinária endógena de frango de corte caipira em diferentes idades ${ }^{1}$

\begin{tabular}{|c|c|c|c|c|}
\hline \multirow{2}{*}{$\begin{array}{l}\text { Idade } \\
\text { (dias) }\end{array}$} & \multicolumn{4}{|c|}{ Matéria seca fecal metabólica e urinária endógena } \\
\hline & (gMS/ave) & $(\mathrm{gMS} / \mathrm{kgPC})$ & $(\%$ de $\mathrm{PC})$ & $\left(\mathrm{gMS} / \mathrm{kgPC}^{0,75}\right)$ \\
\hline 15 & $1,75 \mathrm{~b}$ & $6,41 \mathrm{a}$ & $0,23 \mathrm{a}$ & $8,24 \mathrm{a}$ \\
\hline 45 & $4,32 \mathrm{a}$ & $4,69 \mathrm{~b}$ & $0,06 \mathrm{~b}$ & $7,72 \mathrm{a}$ \\
\hline 75 & $4,03 \mathrm{a}$ & $1,97 \mathrm{c}$ & $0,02 \mathrm{c}$ & $3,69 \mathrm{~b}$ \\
\hline Média & $3,37 \pm 0,24$ & $4,35 \pm 0,23$ & $0,10 \pm 0,007$ & $6,55 \pm 0,339$ \\
\hline $\mathrm{ER}^{1}$ & $\hat{Y}=1,675+0,037 X$ & $\hat{Y}=7,65-0,071 X$ & $\hat{Y}=0,27-0,003 X$ & $\hat{Y}=9,93-0,073 X$ \\
\hline $\mathrm{R}^{2}, \%$ & 65,79 & 98,33 & 89,40 & 83,35 \\
\hline $\mathrm{P}^{2}$ & 0,0001 & 0,0001 & 0,0001 & 0,0001 \\
\hline $\mathrm{CV}^{3}$ & 15,70 & 12,01 & 16,17 & 11,60 \\
\hline
\end{tabular}

a,b,c Médias seguidas de letras distintas, na coluna, diferem estatisticamente pelo teste Student Newman-Keuls a 1\% de probabilidade.

${ }^{1}$ Equação de regressão.

${ }^{2}$ Probabilidade.

${ }^{3}$ Coeficiente de variação, $\%$.

Assim como as aves de corte industrial, as caipiras também apresentaram comportamento inverso quando se relacionou a variável grama de matéria seca com o peso corporal e com o peso metabólico. Diferentemente do sistema anterior (gMS/ave), os sistemas gMS/kgPC, $\%$ de $\mathrm{PC}$ e gMS $/ \mathrm{kgPC}^{0,75}$ exibiram diferenças $(\mathrm{p}<0,01)$ entres as idades experimentais. Para o sistema gMS/ $\mathrm{kgPC}$, entre o $15^{\circ}$ e o $45^{\circ}$ dia de idade a redução observada foi de cerca de $27 \%$. Esse percentual aumentou entre as idades subsequentes, $\left(45^{\circ}\right.$ e $75^{\circ}$ dias de idade), chegando próximo a $58 \%$ de redução, até atingir 1,97 $\mathrm{gMS} / \mathrm{kgPC}$ na última idade avaliada.

$\mathrm{O}$ efeito da idade de frangos caipiras sobre as perdas endógenas de matéria seca metabólica e urinária pode ser visto nas Figuras $2 \mathrm{~A}$ e $2 \mathrm{~B}$ de forma mais nítida, sobretudo quando se relaciona MSfm + MSeu em quilo de peso metabólico. Dessa forma, a excreção por ave diminuiu em $0,073 \mathrm{~g}$ por dia até a última idade experimental (Figura 2B), em detrimento da excreção no sistema grama ave (Figura 2A), que apresentou aumento expressivo até o $45^{\circ}$ dia de idade. Entre essa idade e a subsequente as diferenças não foram percebidas pelo teste de média, e a análise de regressão mostrou um modelo $\left(\hat{\mathrm{Y}}=1,675+0,037 \mathrm{X}, \mathrm{R}^{2}=65,79\right)$ com grau de dispersão considerável, comparado ao modelo anunciado anteriormente com frangos de corte industrial.
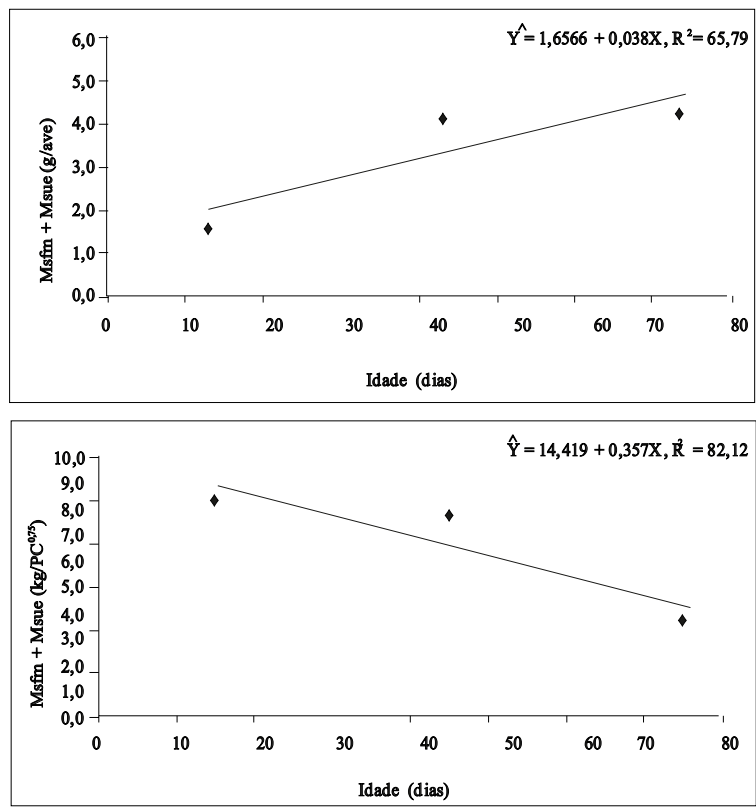

FIGURA 2. Efeito da idade sobre as perdas em matéria seca fecal metabólica e urinária endógena (MSfm + MSeu) de frangos de corte caipira expresso por ave (A) e expresso em peso metabólico (B). 
Esse aumento significativo até o $45^{\circ}$ dia pode ser relacionado à idade fisiológica da ave, ou seja, nessa idade a taxa de crescimento começa a diminuir significativamente (ponto de inflexão da reta) e leva a uma diminuição na taxa metabólica da ave. Ensaios de crescimento com aves dessa categoria e grupo genético foram realizados por SANTOS et al. (2005). Esses autores verificaram, por meio da aplicação da equação de Gompertz, que no ponto de mudança do padrão de aceleração na curva - ou seja, o ponto de inflexão, em que a ave atinge a maior taxa de crescimento, por volta do $46^{\circ}$ dia de vida - a atividade metabólica é intensa.

Esse estado fisiológico pode ter influenciado o aumento expressivo percebido até os 45 dias de idade.
Após esse período, ou seja, na fase subsequente, não se observaram alterações marcantes na excreção metabólica e endógena de matéria seca por ave analisada no presente experimento.

Na Tabela 5 estão apresentados os dados referentes ao nitrogênio metabólico e endógeno (Nfm + Nue) quantificado em frangos de corte industrial em diferentes idades. Os dados obtidos sugerem que as maiores diferenças na excreção do $\mathrm{Nfm}+$ Nue ocorrem nas idades equidistantes 5 e 35 dias, independentemente da forma como está sendo expressa. Desse modo, a ave aumenta sua excreção em 1,112 g por dia.

TABELA 5. Médias e erros-padrões, equação de regressão, probabilidade e coeficiente de variação para os valores das perdas de nitrogênio fecal metabólico e urinário endógeno de frango de corte industrial em diferentes idades ${ }^{1}$

\begin{tabular}{ccccc}
\hline \multirow{2}{*}{$\begin{array}{c}\text { Idade } \\
\text { dias })\end{array}$} & \multicolumn{4}{c}{ Nitrogênio fecal metabólico e urinário endógeno } \\
\cline { 2 - 5 } & $(\mathrm{gN} / \mathrm{ave})$ & $(\mathrm{gN} / \mathrm{kgPC})$ & $(\mathrm{gN}, \%$ de PC $)$ & $(\mathrm{gN} / \mathrm{kgPC}, 75)$ \\
\hline 5 & $1,26 \mathrm{c}$ & $15,22 \mathrm{a}$ & $1,52 \mathrm{a}$ & $14,52 \mathrm{a}$ \\
15 & $2,26 \mathrm{~b}$ & $5,21 \mathrm{~b}$ & $0,52 \mathrm{~b}$ & $7,11 \mathrm{~b}$ \\
25 & $2,38 \mathrm{~b}$ & $2,36 \mathrm{~b}$ & $0,24 \mathrm{~b}$ & $3,70 \mathrm{c}$ \\
35 & $4,96 \mathrm{c}$ & $2,15 \mathrm{~b}$ & $0,21 \mathrm{~b}$ & $\mathrm{c}$ \\
Média & $2,72 \pm 0,17$ & $6,23 \pm 0,85$ & $0,62 \pm 0,08$ & $7,27 \pm 0,83$ \\
$\mathrm{ER}^{1}$ & $\hat{\mathrm{Y}}=0,475+0,112 \mathrm{X}$ & $\hat{\mathrm{Y}}=14,645-0,420 \mathrm{X}$ & $\hat{\mathrm{Y}}=1,467-0,042 \mathrm{X}$ & $\hat{\mathrm{Y}}=14,419-0,357 \mathrm{X}$ \\
$\mathrm{R}^{2}, \%$ & 84,02 & 77,94 & 78,02 & 82,12 \\
$\mathrm{P}^{2}$ & 0,0001 & 0,0001 & 0,0001 & 0,0001 \\
$\mathrm{CV}^{3}$ & 14,39 & 30,62 & 30,72 & 25,66 \\
\hline
\end{tabular}

a,b,c Médias seguidas de letras distintas, na coluna, diferem estatisticamente pelo teste Student Newman-Keuls a 1\% de probabilidade.

${ }^{1}$ Equação de regressão.

${ }^{2}$ Probabilidade.

${ }^{3}$ Coeficiente de variação, \%.

Essa informação, relacionada ao peso corporal, indica que proporcionalmente a maior perda acontece na fase pré-inicial e, após esse período, há redução de cerca de $0,42 \mathrm{~g}$ por dia. Assim, o nitrogênio endógeno e metabólico quantificado na excreta de aves com 15 dias de idade reduz cerca de $66 \%$ quando comparado com a menor idade. Em seguida, de 15 a 25 dias de idade, essa redução diminui para $54 \%$ e tende a estabilizar entre 25 e 35 dias de idade, chegando a apenas $12,5 \%$, de redução.

A perda de nitrogênio no trato digestório tem motivado estudos há bastante tempo. A secreção en- dógena de nitrogênio do trato digestivo de ratos foi investigada pelos pesquisadores TWOMBLY \& MEYER (1961), utilizando ensaios de curta duração. Nesses ensaios, os referidos autores observaram que à medida que aumentava a percentagem de proteína da dieta aumentava o teor de nitrogênio em $0,0459 \mathrm{~g}$, conforme a equação $\hat{Y}=1,31+0,0459 X$. Para o nível máximo de proteína na dieta ao final das 12 horas de alimentação, esses pesquisadores estimaram que cerca de 2,228 g de nitrogênio foram secretados pelo epitélio secretor do trato digestório. Por extrapolação ao consumo zero de proteína, para dieta isenta de proteína, TWOMBLY 
\& MEYER (1961) verificaram algo próximo de 1,31 $\mathrm{g}$ de nitrogênio nas fezes dos animais.

Dados coletados por CHUNG \& BAKER (1992), utilizando galos adultos, revelaram que a perda de nitrogênio fecal metabólico e urinário endógeno $(\mathrm{Nfm}+\mathrm{Nue})$ foi próxima de $0,944 \mathrm{~g}$ por ave. Dados recentes publicados por SILVA et al. (2006) referentes a aves mantidas em jejum demonstraram que um pinto de corte industrial ao $7^{\circ}$ dia de vida perde em média $0,61 \mathrm{~g}$ de $\mathrm{Nfm}+\mathrm{Nue}$; o mesmo frango ao $37^{\circ}$ dia aumenta essa excreção cerca de 3,5 vezes. Esse aumento, nessa mesma proporção, ocorreu nos valores obtidos no presente trabalho.

No entanto, os números absolutos registrados neste estudo, nas diferentes idades, foram superiores aos valores reportados por SILVA et al. (2006). Isso se pauta nos aspectos metodológicos, no que se refere à idade e ao peso da ave submetida ao jejum experimental.

Na Tabela 6 estão apresentados os valores das perdas de nitrogênio pelas aves caipiras. Pode ser visto que a excreção de nitrogênio aumentou com o avanço da idade numa proporção de 0,007 g por dia, sendo o maior aumento entre 15 e 45 dias de idade. A diferença na excreção entre 15 e 45 dias foi de 1,97 vezes - aumento esperado, uma vez que o comprimento do intestino cresce sensivelmente nesse intervalo, conforme foi verificado por FIGUEIREDO et al. (2002).

SANTOS et al. (2005) verificaram que a relação dos intestinos com o peso do animal em jejum corresponde a 3,35\% em frangos de corte caipiras e a 3,8\% nos frangos de corte industriais. Em números relativos essa diferença é estreita, mas em valores absoluto, o frango de corte industrial dispõe de uma maior superfície intestinal para ocorrências de descamações celulares. Essa característica acentuou as diferenças nos resultados obtidos, ao passo que o valor médio verificado para os frangos de corte foi cerca de 4,6 vezes maior que o obtido com as aves caipiras.

TABELA 6. Médias e erros-padrões, equação de regressão, probabilidade e coeficiente de variação das perdas de nitrogênio fecal metabólico e urinário endógeno de frango de corte caipira em diferentes idades ${ }^{1}$

\begin{tabular}{|c|c|c|c|c|}
\hline \multirow{2}{*}{$\begin{array}{l}\text { Idade } \\
\text { (dias) }\end{array}$} & \multirow[b]{2}{*}{ (gN/ave) } & \multirow[b]{2}{*}{$(\mathrm{gN} / \mathrm{kgPC})$} & \multirow[b]{2}{*}{$(\mathrm{gN}, \%$ de $\mathrm{PC})$} & \multirow[b]{2}{*}{$\left(\mathrm{gN} / \mathrm{kgPC}^{0,75}\right)$} \\
\hline & & & & \\
\hline 15 & $0,34 \mathrm{~b}$ & $1,23 \mathrm{a}$ & $0,12 \mathrm{a}$ & $1,58 \mathrm{a}$ \\
\hline 45 & $0,67 \mathrm{a}$ & $0,73 \mathrm{~b}$ & $0,07 \mathrm{~b}$ & $1,20 \mathrm{~b}$ \\
\hline 75 & $0,78 \mathrm{a}$ & $0,38 \mathrm{c}$ & $0,04 \mathrm{c}$ & $0,71 \mathrm{c}$ \\
\hline Média & $0,59 \pm 0,55$ & $0,78 \pm 0,049$ & $0,08 \pm 0,005$ & $1,16 \pm 0,077$ \\
\hline $\mathrm{ER}^{1}$ & $\hat{\mathrm{Y}}=0,267+0,007 \mathrm{X}$ & $\hat{\mathrm{Y}}=1,41-0,013 \mathrm{X}$ & $\hat{\mathrm{Y}}=0,139-0,0013 \mathrm{X}$ & $\hat{\mathrm{Y}}=1,81-0,014 \mathrm{X}$ \\
\hline $\mathrm{R}^{2}, \%$ & 92,05 & 98,76 & 98,42 & 99,64 \\
\hline $\mathrm{P}^{2}$ & 0,001 & 0,0001 & 0,0001 & 0,077 \\
\hline $\mathrm{CV}^{3}$ & 20,52 & 14,09 & 14,62 & 14,81 \\
\hline
\end{tabular}

${ }^{\text {a,b,c }}$ Médias seguidas de letras distintas, na coluna, diferem estatisticamente pelo teste Student Newman-Keuls a $1 \%$ de probabilidade.

${ }^{1}$ Equação de regressão.

${ }^{2}$ Probabilidade.

${ }^{3}$ Coeficiente de variação, $\%$.

A fim de atender às necessidades corporais com o avançar da idade, para desenvolver e aprimorar a capacidade digestiva-absortiva, o crescimento dos intestinos obriga a ocorrência de modificações no epitélio, especialmente no jejuno e íleo, no que se refere ao número e ao volume dos enterócitos (MORAN JUNIOR, 1985; UNI et al., 1995). Esses fenômenos resultam em descamações diárias que, somadas à flora do intestino grosso e à mobilização de reservas corporais para atender à exigência de mantença no período de jejum, podem ter exercido efeito direto sobre o quantitativo de nitrogênio presente nas perdas endógenas e metabólicas, mais intensas frango de corte industrial. 
No estado de jejum prolongado, o nível de aminoácidos circulantes na corrente sanguínea é alto. Esse, por sua vez, são direcionados às células do tecido hepático, onde sofrem oxidação completa ou mesmo podem ser convertidos em glicose, bem como em corpos cetônicos (BROSNAN, 2000; MALHEIROS, 2008). Nesse momento, o aminoácido alanina desempenha papel crucial como importante substrato da gliconeogênese (STRAYER, 1995; NELSON \& COX, 2000). Segundo esses estudiosos, as proteínas do tecido muscular são utilizadas para essa finalidade, mas num período curto, pois tende a ser preservada, uma vez que sua reserva é limitada.

Assim, a mobilização de reservas é a principal fonte para síntese de glicose hepática durante períodos de jejum prolongado; os precursores prioritários são aminoácidos, advindos principalmente do músculo esquelético. Em outro momento, o glicerol proveniente da mobilização das reservas de gordura, triglicerídeos do tecido adiposo e o lactato oriundo das hemáceas constituem os artifícios utilizados pelo metabolismo do animal durante a coleta de dados utilizando essa metodologia, com aves em jejum.

Dessa forma, aves em regime ad libitum utilizam artifícios metabólicos diferentes dos anunciados anteriormente. Assim, as perdas endógenas e metabólicas tendem a ser diferentes, uma vez que o nitrogênio presente na urina (em aves alimentadas) não vem da mobilização de reservas, e sim de catabolismo dos aminoácidos provindos da dieta.

Informações dessa natureza, segundo WARPECHOWSKI et al. (2006), têm sido motivo de discussão em relação ao sistema de energia metabolizável verdadeira, uma vez que no cálculo do balanço de nitrogênio verdadeiro se computa a perda endógena $\mathrm{e}$ metabólica desse componente. Dessa maneira, o balanço é influenciado diretamente pelo método utilizado na coleta do dado e, consequentemente, nos valores de energia metabolizável.

Nas Tabelas 7 e 8 estão apresentadas as perdas de energia seca fecal metabólica e urinária endógena $($ Efm + Eue $)$ em frangos de corte industrial e caipira em função da idade. A excreção de energia fecal metabólica e urinária endógena por parcela mostrou-se diferente apenas na fase pré-inicial, não havendo mais alteração nas fases subsequentes, conforme a análise estatística. No entanto, é prudente salientar que alto coeficiente de variação fez que a diferença mínima significativa fosse superior a $43 \mathrm{kcal}$ nas demais idades.

Os modelos de equações gerados para a linhagem industrial foram lineares, enquanto as aves caipiras se adequaram aos modelos quadráticos. A Efm + Eue verificada para cada frango de corte caipira assumiu o valor máximo de $15,7 \mathrm{kcal}$ aos 57 dias de idade, conforme o modelo $\hat{\mathrm{Y}}=-2,79+0,65 \mathrm{X}-0,0057 \mathrm{X}^{2}$. A excreção máxima das aves caipiras foi inferior ao menor valor obtido para os frangos de corte industrial, que segundo a análise de regressão aumenta conforme a idade em 1,86 kcal por dia.

A energia metabolizável verdadeira (EMV) é obtida pela diferença entre a energia consumida (Econ) e a energia excretada (Eexc), corrigida para energia fecal metabólica e urinária endógena $(\mathrm{Efm}+\mathrm{Eeu})$. Assim, a fórmula é: EMV $=$ Econ - Eexc - MSfm + MSeu. Admitindo-se consumo de ração e excreção constantes, variando-se apenas o valor da Efm + Eeu aos 5 dias (208 kcal) e a média (308 kcal) entre os 15 e os 35 dias idade, no cálculo da EMV, pode se especular que a adoção do valor médio de $308 \mathrm{kcal} /$ parcela pode superestimar metabolizabilidade de uma ração para pintos com 5 dias de idade em mais de $8 \%$, considerando como fonte de variação unicamente a Efm + Eeu para pintos de corte industrial.

Isso se deve ao fato de que, em baixos níveis de consumo, as perdas são proporcionalmente maiores e resultam em diminuição dos valores estimados para energia metabolizável aparente (EMA) e aparente corrigida (EMAn), segundo LIMA et al. (1989).

SILVA et al. (2006) ressaltam que efeitos derivados a partir do baixo consumo por aves experimentais são mais pronunciados quando se utiliza a metodologia de alimentação forçada preconizada por SIBBALD (1976). Resultados de experimentos conduzidos por LIMA et al. (1989) apontaram para valores significativamente diferentes quando comparados aos valores de EMV vs EMA e EMVn vs EMAn, evidenciando o efeito das perdas endógenas e metabólicas em baixos níveis de ingestão. Em níveis normais de consumo, as perdas endógenas e metabólicas são pequenas em relação à excreção de energia proveniente do alimento e tendem a demonstrar pouca influência nos valores de EMA e EMAn (LIMA et al., 1989).

Embora alguns trabalhos tenham visto que matematicamente não há diferença nos valores de energia 
metabolizável utilizando a metodologia tradicional de coleta total com aves em regime ad libitum, a natureza dos compostos excretados e o perfil metabólico das aves em jejum são diferentes dos encontrados nas aves sob alimentação. Assim, a correção feita nos valores de
EMV com informações das perdas endógenas e metabólicas de aves mantidas em jejum não representa o real valor da energia do alimento. Além desses aspectos, elas ainda diferem com a idade e o peso metabólico, seja frango industrial ou caipira.

TABELA 7. Médias e erros-padrões, equação de regressão, probabilidade e coeficiente de variação dos valores das perdas de energia fecal metabólica e urinária endógena de frango de corte industrial em diferentes idades ${ }^{1}$

\begin{tabular}{cccc}
\hline \multirow{2}{*}{$\begin{array}{c}\text { Idade } \\
\text { (dias) }\end{array}$} & \multicolumn{3}{c}{ Energia fecal metabólica e urinária endógena } \\
\cline { 2 - 4 } 5 & $20,87 \mathrm{~d}$ & $(\mathrm{kcal} / \mathrm{kgPC})$ & $\left.(\mathrm{kcal} / \mathrm{kgPC})^{0,75}\right)$ \\
\hline 15 & $36,04 \mathrm{c}$ & $251,65 \mathrm{a}$ & $240,07 \mathrm{a}$ \\
25 & $55,22 \mathrm{~b}$ & $83,11 \mathrm{~b}$ & $113,41 \mathrm{~b}$ \\
35 & $76,54 \mathrm{a}$ & $54,93 \mathrm{c}$ & $86,09 \mathrm{c}$ \\
Média & $47,17 \pm 1,92$ & $33,15 \mathrm{c}$ & $57,78 \mathrm{~d}$ \\
$\mathrm{ER}^{1}$ & $\hat{\mathrm{Y}}=9,93+1,86 \mathrm{X}$ & $105,71 \pm 9,22$ & $124,34 \pm 8,81$ \\
$\mathrm{R}^{2}, \%$ & 99,45 & $\hat{\mathrm{Y}}=242,5-6,8 \mathrm{X}$ & $\hat{\mathrm{Y}}=239,1-5,74 \mathrm{X}$ \\
$\mathrm{P}^{2}$ & 0,0001 & 78,82 & 84,94 \\
$\mathrm{CV}^{3}$ & 9,14 & 0,0001 & 0,0001 \\
\end{tabular}

a,b,c Médias seguidas de letras distintas, na coluna, diferem estatisticamente pelo teste Student Newman-Keuls a $1 \%$ de probabilidade. ${ }^{1}$ Equação de regressão.

${ }^{2}$ Probabilidade.

${ }^{3}$ Coeficiente de variação, \%.

TABELA 8. Médias e erros-padrões, equação de regressão, probabilidade e coeficiente de variação dos valores das perdas de energia seca fecal metabólica e urinária endógena de frango de corte caipira em diferentes idades ${ }^{1}$

\begin{tabular}{cccc}
\hline \multirow{2}{*}{$\begin{array}{c}\text { Idade } \\
\text { (dias) }\end{array}$} & \multicolumn{3}{c}{ Energia fecal metabólica e urinária endógena } \\
\cline { 2 - 4 } & $(\mathrm{kcal} / \mathrm{ave})$ & $(\mathrm{kcal} / \mathrm{kgPC})$ & $(\mathrm{kcal} / \mathrm{kgPC}, 75)$ \\
\hline 15 & $5,67 \mathrm{~b}$ & $20,77 \mathrm{a}$ & $26,70 \mathrm{a}$ \\
75 & $15,09 \mathrm{a}$ & $16,40 \mathrm{~b}$ & $27,01 \mathrm{a}$ \\
$\mathrm{Média}$ & $13,63 \mathrm{a}$ & $6,66 \mathrm{c}$ & $12,47 \mathrm{~b}$ \\
$\mathrm{ER}^{1}$ & $11,47 \pm 0,83$ & $14,61 \pm 0,71$ & $22,06 \pm 1,07$ \\
$\mathrm{R}^{2}$ & $\hat{\mathrm{Y}}=-2,79+0,65 \mathrm{X}-0,0057 \mathrm{X}^{2}$ & $\hat{\mathrm{Y}}=25,08-0,227 \mathrm{X}$ & $\hat{\mathrm{Y}}=32,62-0,229 \mathrm{X}$ \\
$\mathrm{P}^{2}$ & 99,29 & 95,42 & 73,41 \\
$\mathrm{CV}^{3}$ & 0,0001 & 0,0001 & 0,0001 \\
\hline
\end{tabular}

a,b,c Médias seguidas de letras distintas, na coluna, diferem estatisticamente pelo teste Student Newman-Keuls a 1\% de probabilidade.

${ }^{1}$ Equação de regressão.

${ }^{2}$ Probabilidade.

${ }^{3}$ Coeficiente de variação, $\%$.

\section{CONCLUSÃO}

As perdas endógenas e metabólicas de frangos de corte industrial e caipira diminuíram com o avanço da idade, sendo mais acentuadas para frangos de corte industrial. 


\section{REFERÊNCIAS}

BLAXTER, K. Energy metabolism in animals and man. Cambridge: Cambridge University Press, 1989. 336 p.

BORGES, F. M. O.; ROSTAGNO, H. S.; SAAD, C. E. P.; RODRIGUEZ, N. M.; TEIXEIRA, E. A.; LARA, L. B.; MENDES, W. S., ARAÚJO, V. L. Comparação de métodos de avaliação dos valores energéticos do grão de trigo e seus subprodutos para frangos de corte. Arquivo Brasileiro Medicina Veterinária e Zootecnia, v. 55 , n. 6 , p. $710-721,2003$.

BROSNAN, .J. T. Glutamate, at the interface between amino acid and carbohydrate metabolism. Journal of Nutrition, v. 130, n. 4, p. 988S-990S, 2000.

CHUNG, T. K.; BAKER, D. H. Apparent and true amino acid digestibility of a crystalline amino acid mixture and of casein: comparison of values obtained with ileal-cannlated pigs and cecectomized cockerels. Journal Animal Science, v. 70, n. 12, p. 3.781-3.790, 1992.

DALE, N. M.; FULLER, H. L. Applicability of the metabolizable energy system in practical feed formulation. Poultry Science, v. 61, n. 2, p. 351-356, 1982.

FERREIRA, D. F. Programa SISVAR. Sistema de análise de variância. Versão 4.6. Lavras: Universidade Federal de Lavras, 2003.

FIGUEIREDO, E. P. A.; ROSA, O. S.; OMM, E. R.; BOFF, J. A.; BASSI, L. Peso e tamanho dos órgãos de diferentes linhagens de frangos de corte. Revista Brasileira de Ciência Avícola, v. 4, supl. 4, p. 95, 2002.

FREITAS, E. R.; SAKOMURA, N. K.; EZEQUIEL, J. M. B.; NEME, R.; MENDONÇA, M. O. Energia metabolizável de alimentos na formulação de ração para frangos de corte. Pesquisa Agropecuária Brasileira, v. 41, n. 1, p. 107-115, 2006.

LIMA, L. I.; SILVA, J. D. da.; ROSTAGNO, S. H. TARFURI, M. L. Composição química e valores energéticos de alguns alimentos determinados com pintos e galos, utilizando duas metodologias. Revista Brasileira de Zootecnia, v. 18, n. 6, p. 546-556, 1989.

MALHEIROS, S. V. P. Integração metabólica nos períodos pós-prandial e de jejum. Revista Brasileira de Ensino de Bioquímica e Biologia Molecular. Disponível em: $<$ http://www. bdc.ib.unicamp.br/rbebbm/>. Acesso em: 22 jul. 2008.

MORAN JR. E. T. Digestion and absorption of carbohydrates in fowl and events through perinatal development. Journal of Nutrition, v. 115, n. 5, p. 665-674, 1985.
NELSON, D. L.; COX, M. M.; Lehninger, principles of biochemistry. 3. ed. Worths Publishers: New York, 2000. p. 949.

ROSTAGNO, H. S.; ALBINO, L. F. T.; DONZELE, J. L.; GOMES, P. C.; OLIVEIRA, R. F. M.; LOPES, D. C.; FERREIRA, A. S.; BARRETO, S. L. T. Tabelas brasileiras para aves e suínos (Composição de alimentos e exigências nutricionais). 2. ed. Viçosa: Edutira UFV, 2005. 186 p.

SANTOS A. L. dos.; SAKOMURA, N. K.; FREITAS, E. R.; FORTES, C. M. L. S.; CARRILHO, E. N. V. M.; FERNANDES, J. B. K. Estudo do crescimento, desempenho, rendimento de carcaça e qualidade de carne de três linhagens de frango de corte. Revista Brasileira de Zootecnia, v. 34, n. 5, p. 1.589-1.598, 2005.

SIBBALD, I. R. The true metabolizable energy values of several feedstuffs measured with roosters, laying hens, turkeys and broiler hens. Poultry Science, v. 55, n. 4, p. 1.459-1.463, 1976.

SIBBALD, I. R. Metabolic plus endogenous energy and nitrogen loses of adult cockerels the correction used in the bioassay for true metabolizable energy. Poultry Science, v. 60, n. 4, p. 805-811, 1981a.

SIBBALD, I. R. Metabolic plus endogenous energy excretion by fowl. Poultry Science, v. 60, n. 12, p. 2.672-2.677, 1981 b.

SIBBALD, I. R.; PRICE, K. The metabolic and endogenous energy losses of adult roosters. Poultry Science, v. 57, n. 2, p. 556-557, 1978.

SILVA, D. J.; QUEIROZ, A. C. Análise de alimentos (métodos químicos e biológicos). 3. ed. Viçosa: Editora UFV, 2002. 235 p.

SILVA, E. P.; RABELLO, C. B. V.; LIRA, R. C.; FARIAS FILHO, R. V.; ALBUQUERQUE, C. S.; SILVA, da. T. Estimativas das perdas endógenas e metabólicas em frangos de corte. Revista Brasileira de Ciências Agrárias, v. 1, n. único, p. 115-121, 2006.

STRAYER, L. Biochemistry. 4. ed. New York: W.H. Freeman, 1995.

TWOMBLY, J.; MEYER, J. H. Endogenous nitrogen secretions into the digestive tract. Journal of Nutrition, v. 74, n. 4, p. 453-460, 1961.

UNI, Z.; NOY, Y.; SKLAN, D. Post-hatch changes in morphology and function of the small intestine in heavy and light strain chicks. Poultry Science, v. 74, n. 10, p. 1.622-1.629, 1995.

WARPECHOWSKI, M. B.; KESSLER, A. M. POPHAL, S.; EBERT, A.; RIBEIRO, A. M. L. Digestibilidade ideal verdadeira da proteína em frangos de corte sob dietas com diferentes níveis de proteína bruta. Acta Scientiarum Animal Science, v. 28, n. 3, p. 281-287, 2006. 